\title{
A space-time coding approach for RFID MIMO systems
}

\author{
Feng Zheng* and Thomas Kaiser
}

\begin{abstract}
This paper discusses the space-time coding (STC) problem for RFID MIMO systems. First, a mathematical model for this kind of system is developed from the viewpoint of signal processing, which makes it easy to design the STC schemes. Then two STC schemes, namely Scheme I and Scheme II, are proposed. Simulation results illustrate that the proposed approaches can greatly improve the symbol-error rate (SER) or bit-error rate (BER) performance of RFID systems, compared to the non space-time encoded RFID system. The SER/BER performance for Scheme I and Scheme II is thoroughly compared. It is found that Scheme II with the innate real-symbol constellation yields better SER/BER performance than Scheme I. Some design guidelines for RFID-MIMO systems are pointed out.
\end{abstract}

\section{Introduction}

Radio frequency identification (RFID) is a contactless, usually short distance, wireless data transmission and reception technique for identification of objects. It is believed that RFID can substitute, in the not-far future, the widely used optical barcode technology due to the limitations of the latter in i) the barcode cannot read nonline-of-sight (NLOS) tag; ii) each barcode needs personal care to be read; and iii) limited information-carrying ability of the barcode. Currently, a single antenna is usually used at the reader and tag of RFID in the market. However, RFID research community recently started to pay attention on using multiple antennas at either the reader side or the tag side $[1,2]$. The reason is that using multiple antennas is an efficient approach to increasing the coverage of RFID, solving the NLOS problem, improving the reliability of data communications between the reader and tag, and thus further extending the information-carrying ability of RFID. Besides, some advanced technology in multiple transmit and receive antennas (MIMO) can be used to solve the problem of detecting multiple objects simultaneously, see e.g., [3].

There have been several studies about RFID-MIMO. In general, these studies are somehow scattered in different topics. It is difficult to find the logical relationship among these studies. Therefore, the state of the art of the

*Correspondence: feng.zheng@uni-due.de

Institute of Digital Signal Processing, University of Duisburg-Essen, 47057 Duisburg, Germany studies will be reviewed in a large degree in a chronological order. The work [4] first showed the idea of using multiple antennas at the reader for both transmission and reception. In [1], the authors first proposed to use multiple antennas at the tag and showed the performance gain by equipping multiple antennas at the reader (for both transmission and reception) and the tag. In [5], the multipath fading for both single-antenna based RFID channel and RFID-MIMO channel was measured and compared. The improvement on the fading depth by using MIMO can be clearly seen from the measured power distribution (see, e.g., Figure Ten therein). In [6], the authors first proposed to apply the Alamouti space-time coding (STC) technique, which is now popularly used in wireless communication systems, to the RFID systems. The reference [6] presented a closed-form expression for the bit-error rate (BER) of the RFID system with the nonecoherent frequency shift keying modulation and multiple transmit antennas at the tag and single transmit/receive antenna at the reader, where the double Rayleigh fading is assumed at the forward and backward links. In [7], the interrogation range of ultrahigh-frequency-band (UHFband) RFID with multiple transmit/receive antennas at the reader and single antenna at the tag was analyzed, where the forward and backward channels are assumed to take the Nakagami- $m$ distribution. In [3], the blind source separation technique in antenna array was used to solve the multiple tag identification problem, where the reader is equipped with multiple antennas. The work

\section{照 Springer}

(c) 2012 Zheng and Kaiser; licensee Springer. This is an Open Access article distributed under the terms of the Creative Commons Attribution License (http://creativecommons.org/licenses/by/2.0), which permits unrestricted use, distribution, and reproduction in any medium, provided the original work is properly cited. 
[8] applied the maximal ratio combining technique to the RFID receiver, where the channel of the whole chain, including forward link, backscattering coefficient, and backward link, was estimated and used as the weighting coefficient for the combining branches. In [9], a prototype for the RFID-MIMO in the UHF-band was reported. In [10], both MIMO-based zero-forcing and minimummean-square-error receivers were used to deal with the multiple-tag identification problem, where the channel of the whole chain was estimated, similar to the approach in [8]. It is reported in [11] that four antennas are fabricated in a given fixed surface at the reader. The measurement results showed that an increase of $83 \%$ in area gave a $300 \%$ increase in available power to turn on a given tag load and the operational distance of the powered device is increased to $100 \mathrm{~cm}$ by the four-antenna setup from roughly $40 \mathrm{~cm}$ for the single-antenna setup. The result in [11] suggests that the MIMO technique can be very promising to the RFID technology.

In the aforementioned reports, the Alamouti STC technique has been shown to be able to extend to RFID-MIMO systems. However, it can only apply to the case where the tag has two antennas. Since implementing four antennas at the tag have been shown to be possible in experiments, it is necessary to investigate the possibility of applying other STC techniques to RFID-MIMO systems. In this paper, we will study how to apply the real orthogonal design (ROD) technique, proposed by Tarokh et al. in [12], to RFID-MIMO systems. This technique is suitable for the case where the tag is equipped up to eight antennas, which should be sufficient for the RFID technology in the near future.

The paper is organized as follows. A modified MIMORFID channel model will be developed in Section "Channel Modeling of RFID MIMO Wireless Systems". The ROD in [12] and the companion of the ROD (CROD) proposed in [13] are briefly introduced in Section "A Space-Time Coding Scheme for RFID MIMO Systems". Two space-time decoding approaches for RFID MIMO systems will be discussed in Section "Two Space-Time Decoding Approaches for RFID MIMO Systems". Section "Simulation Results" presents the simulation results and discussions, and Section "Conclusions" concludes the paper.

\section{Channel Modeling of RFID MIMO Wireless Systems}

In this paper our discussion is confined only on narrowband RFID systems. The block diagram of the RFID MIMO system is illustrated in Figure 1, where both the reader and tag are equipped with multiple antennas.

In terms of equation (1) of [1], the narrowband RFID MIMO wireless channel can be expressed as

$$
\mathbf{y}(t)=\mathbf{H}^{\mathrm{b}} \mathbf{S}(t) \mathbf{H}^{\mathrm{f}} \mathbf{x}(t)+\mathbf{n}(t),
$$

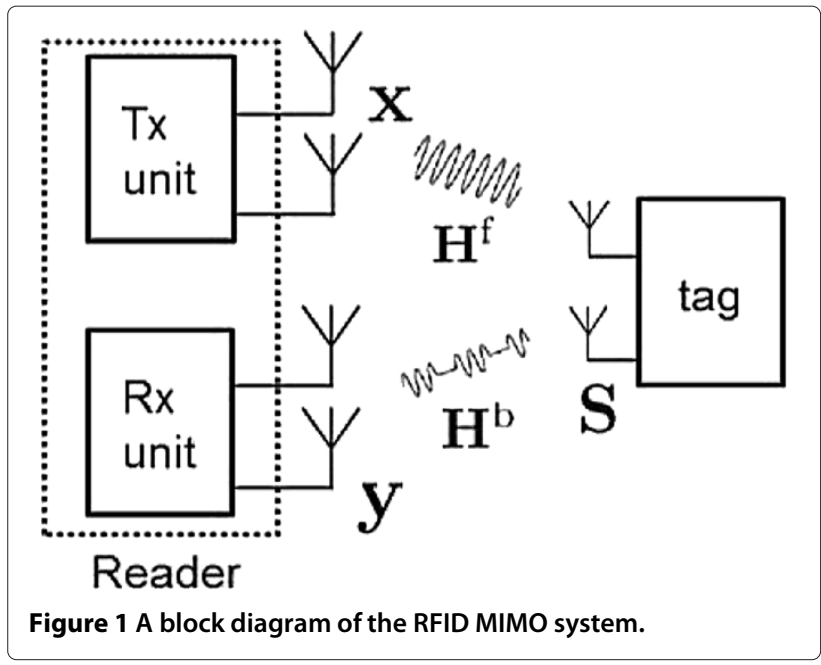

where the reader and tag are equipped with $N_{\text {rd }}$ and $N_{\text {tag }}$ antennas, respectively, $\mathbf{x}$ (an $N_{\mathrm{rd}} \times 1$ vector) is the transmitted signal at the reader, $\mathbf{y}$ (an $N_{\text {rd }} \times 1$ vector) is the received signal at the reader, $\mathbf{n}$ is the receiver noise, $\mathbf{H}^{\mathrm{f}}$ (an $N_{\text {tag }} \times N_{\text {rd }}$ matrix) is the channel matrix from the reader to the tag, $\mathbf{H}^{\mathrm{b}}$ (an $N_{\text {rd }} \times N_{\text {tag }}$ matrix) is the channel matrix from the tag to the reader, and $\mathbf{S}$ is the backscattering matrix, which is also called signaling matrix. It is assumed that the $N_{\text {rd }}$ antennas at the reader are used for both reception and transmission. This assumption is just for brevity of the notation. It is straightforward to extend the approach presented in this paper to the case where the reader has different numbers of antennas for reception and transmission. The channels $\mathbf{H}^{\mathrm{f}}$ and $\mathbf{H}^{\mathrm{b}}$ are assumed to be complex Gaussian distributed, $\mathbf{H}^{\mathrm{f}}$ and $\mathbf{H}^{\mathrm{b}}$ are mutually independent, and all the entries of either $\mathbf{H}^{\mathrm{f}}$ or $\mathbf{H}^{\mathrm{b}}$ are independent of each other. It is also assumed that $\operatorname{Re}\left(\mathbf{H}^{\mathrm{f}}\right)$, $\operatorname{Im}\left(\mathbf{H}^{\mathrm{f}}\right), \operatorname{Re}\left(\mathbf{H}^{\mathrm{b}}\right), \operatorname{Im}\left(\mathbf{H}^{\mathrm{b}}\right)$ are mutually independent and of the same distribution.

In most general case where the modulated backscatter signals at the tag are transferred between the antennas, the signaling matrix $\mathbf{S}$ is a full matrix [1]. However no application of the full signalling matrix has been identified up to now [1]. Therefore, we will consider the situation where the RF tag antennas modulate backscatter with different signals and no signals are transferred between the antennas. In this case, the signaling matrix is a diagonal matrix [1]

$\mathbf{S}(t)=\operatorname{diag}\left\{\Gamma_{1}(t), \Gamma_{2}(t) \ldots, \Gamma_{N_{\mathrm{tag}}}(t)\right\}$ with $\left|\Gamma_{i}(t)\right| \leq 1$,

where $\Gamma_{i}(t)$ is the backscattering coefficient of $i$ th antenna at the tag. The $i$ th tag identity (ID) is contained in the coefficient $\Gamma_{i}(t)$.

Note that in the RFID system, the transmitted signal $\mathbf{x}$ is mainly used to carry the transmit power, while the information data (i.e., tag ID) is carried out by $\mathbf{S}$. Therefore, the 
central issue for the RFID is to decode $\Gamma_{1}, \ldots, \Gamma_{N_{\text {tag }}}$ from the received signal. Next we transform equation (1) to the conventional form in signal processing. Let us define

$$
\boldsymbol{\gamma}(t)=\left[\begin{array}{c}
\Gamma_{1}(t) \\
\Gamma_{2}(t) \\
\vdots \\
\Gamma_{N_{\mathrm{tag}}}(t)
\end{array}\right], \quad \mathbf{H}^{\mathrm{f}}=\left[\begin{array}{c}
\mathbf{H}_{1}^{\mathrm{f}} \\
\mathbf{H}_{2}^{\mathrm{f}} \\
\vdots \\
\mathbf{H}_{N_{\mathrm{tag}}}^{\mathrm{f}}
\end{array}\right] .
$$

Then equation (1) can be rewritten as

$$
\begin{aligned}
& \mathbf{y}(t)=\mathbf{H}^{\mathrm{b}} \text { diag }\left\{\Gamma_{1}(t), \Gamma_{2}(t) \ldots, \Gamma_{N_{\mathrm{tag}}}(t)\right\} \mathbf{H}^{\mathrm{f}} \mathbf{x}(t)+\mathbf{n}(t) \\
& =\mathbf{H}^{\mathrm{b}} \operatorname{diag}\{1,0, \ldots, 0\} \mathbf{H}^{\mathrm{f}} \mathbf{x}(t) \Gamma_{1}(t) \\
& +\mathbf{H}^{\mathrm{b}} \text { diag }\{0,1, \ldots, 0\} \mathbf{H}^{\mathrm{f}} \mathbf{x}(t) \Gamma_{2}(t)+\cdots \\
& +\mathbf{H}^{\mathrm{b}} \text { diag }\{0,0, \ldots, 1\} \mathbf{H}^{\mathrm{f}} \mathbf{x}(t) \Gamma_{N_{\mathrm{tag}}}(t)+\mathbf{n}(t) \\
& =\mathbf{H}^{\mathrm{b}}\left[\begin{array}{c}
\mathbf{H}_{1}^{\mathrm{f}} \\
\mathbf{0} \\
\vdots \\
\mathbf{0}
\end{array}\right] \mathbf{x}(t) \Gamma_{1}(t)+\mathbf{H}^{\mathrm{b}}\left[\begin{array}{c}
\mathbf{0} \\
\mathbf{H}_{2}^{\mathrm{f}} \\
\vdots \\
\mathbf{0}
\end{array}\right] \mathbf{x}(t) \Gamma_{2}(t)+\cdots \\
& +\mathbf{H}^{\mathrm{b}}\left[\begin{array}{c}
\mathbf{0} \\
\mathbf{0} \\
\vdots \\
\mathbf{H}_{N_{\mathrm{tag}}}^{\mathrm{f}}
\end{array}\right] \mathbf{x}(t) \Gamma_{N_{\mathrm{tag}}}(t)+\mathbf{n}(t) \\
& =\mathbf{H}^{\mathrm{b}}\left[\begin{array}{cccc}
\mathbf{H}_{1}^{\mathrm{f}} \mathbf{x}(t) & 0 & \cdots & 0 \\
0 & \mathbf{H}_{2}^{\mathrm{f}} \mathbf{x}(t) & \cdots & 0 \\
\vdots & \vdots & \ddots & \vdots \\
0 & 0 & \cdots & \mathbf{H}_{N_{\mathrm{tag}}}^{\mathrm{f}} \mathbf{x}(t)
\end{array}\right] \\
& \times\left[\begin{array}{c}
\Gamma_{1}(t) \\
\Gamma_{2}(t) \\
\vdots \\
\Gamma_{N_{\text {tag }}}(t)
\end{array}\right]+\mathbf{n}(t) \\
& =\mathbf{H}^{\mathrm{b}} \breve{\mathbf{H}}(t) \boldsymbol{\gamma}(t)+\mathbf{n}(t),
\end{aligned}
$$

where

$$
\breve{\mathbf{H}}(t):=\left[\begin{array}{cccc}
\mathbf{H}_{1}^{\mathrm{f}} \mathbf{x}(t) & 0 & \cdots & 0 \\
0 & \mathbf{H}_{2}^{\mathrm{f}} \mathbf{x}(t) & \cdots & 0 \\
\vdots & \vdots & \ddots & \vdots \\
0 & 0 & \cdots & \mathbf{H}_{N_{\mathrm{tag}}^{\mathrm{f}}}^{\mathrm{x}} \mathbf{x}(t)
\end{array}\right] .
$$

Equation (3) converts the original system model (1) to the conventional form in signal processing: the signal to be estimated or decoded is packed in a vector, whose entries are independent of each other.

\section{A Space-Time Coding Scheme for RFID MIMO Systems}

Let us first review the real orthogonal design proposed by Tarokh et al. in [12].

Definition 1. [12] A real orthogonal design $\mathcal{G}$ of size $m$ is an $m \times k$ matrix with entries $0, \pm \mathcal{S}_{1}, \pm \mathcal{S}_{2}, \ldots, \pm \mathcal{S}_{k}$ such that $\mathcal{G G}^{T}=\mathbf{D}$, where $\mathbf{D}$ is a diagonal matrix with diagonal entries being $\mathbf{D}_{i i}=l_{i 1} \mathcal{S}_{1}^{2}+l_{i 2} \mathcal{S}_{2}^{2}+\cdots+l_{i k} \mathcal{S}_{k}^{2}$, $i=1,2, \ldots, m$, and the coefficients $l_{i 1}, l_{i 2}, \ldots, l_{i k}$ are strictly positive integers.

In some cases, we need to explicitly specify the arguments of $\mathcal{G}$. In these cases, the ROD will be denoted as $\mathcal{G}\left(\mathcal{S}_{1}, \mathcal{S}_{2}, \ldots, \mathcal{S}_{k}\right)$, where $\mathcal{S}_{1}, \mathcal{S}_{2}, \ldots, \mathcal{S}_{k}$ are the arguments of $\mathcal{G}$.

The construction of general RODs can be found in [12]. For completeness, the RODs for the cases of $m=$ 2, 3, 4, denoted as $\mathcal{G}^{(2)}, \mathcal{G}^{(3)}, \mathcal{G}^{(4)}$ respectively, are listed as follows:

$$
\begin{aligned}
\mathcal{G}^{(2)}= & {\left[\begin{array}{rr}
\mathcal{S}_{1} & -\mathcal{S}_{2} \\
\mathcal{S}_{2} & \mathcal{S}_{1}
\end{array}\right], } \\
\mathcal{G}^{(3)}= & {\left[\begin{array}{rrrr}
\mathcal{S}_{1} & -\mathcal{S}_{2} & -\mathcal{S}_{3} & -\mathcal{S}_{4} \\
\mathcal{S}_{2} & \mathcal{S}_{1} & \mathcal{S}_{4} & -\mathcal{S}_{3} \\
\mathcal{S}_{3} & -\mathcal{S}_{4} & \mathcal{S}_{1} & \mathcal{S}_{2}
\end{array}\right], } \\
\mathcal{G}^{(4)}= & {\left[\begin{array}{rrrr}
\mathcal{S}_{1} & -\mathcal{S}_{2} & -\mathcal{S}_{3} & -\mathcal{S}_{4} \\
\mathcal{S}_{2} & \mathcal{S}_{1} & \mathcal{S}_{4} & -\mathcal{S}_{3} \\
\mathcal{S}_{3} & -\mathcal{S}_{4} & \mathcal{S}_{1} & \mathcal{S}_{2} \\
\mathcal{S}_{4} & \mathcal{S}_{3} & -\mathcal{S}_{2} & \mathcal{S}_{1}
\end{array}\right] . }
\end{aligned}
$$

For the construction of $\mathcal{G}^{(5)}, \ldots, \mathcal{G}^{(8)}$, readers are referred to [12].

To formulate the decoding algorithm for the ROD, let us define the companion of the ROD as follows.

Definition 2. A companion of a real orthogonal design $\mathcal{G}\left(\mathcal{S}_{1}, \mathcal{S}_{2}, \ldots, \mathcal{S}_{k}\right)$, denoted as $\mathcal{G}_{c}\left(\alpha_{1}, \alpha_{2}, \ldots, \alpha_{m}\right)$, is a matrix satisfying the following equation

$$
\begin{aligned}
& {\left[\begin{array}{llll}
\alpha_{1} & \alpha_{2} & \ldots & \alpha_{m}
\end{array}\right] \mathcal{G}\left(\mathcal{S}_{1}, \mathcal{S}_{2}, \ldots, \mathcal{S}_{k}\right)=\left[\mathcal{S}_{1} \mathcal{S}_{2} \ldots \mathcal{S}_{k}\right]} \\
& \times \mathcal{G}_{c}\left(\alpha_{1}, \alpha_{2}, \ldots, \alpha_{m}\right) \text {. }
\end{aligned}
$$

For the RODs as shown in equations (4)-(6), their CRODs are

$$
\begin{aligned}
\mathcal{G}_{c}^{(2)} & =\left[\begin{array}{rr}
\alpha_{1} & \alpha_{2} \\
\alpha_{2} & -\alpha_{1}
\end{array}\right], \\
\mathcal{G}_{c}^{(3)} & =\left[\begin{array}{rrrr}
\alpha_{1} & \alpha_{2} & \alpha_{3} & 0 \\
\alpha_{2} & -\alpha_{1} & 0 & \alpha_{3} \\
\alpha_{3} & 0 & -\alpha_{1} & -\alpha_{2} \\
0 & -\alpha_{3} & \alpha_{2} & -\alpha_{1}
\end{array}\right],
\end{aligned}
$$




$$
\mathcal{G}_{c}^{(4)}=\left[\begin{array}{rrrr}
\alpha_{1} & \alpha_{2} & \alpha_{3} & \alpha_{4} \\
\alpha_{2} & -\alpha_{1} & -\alpha_{4} & \alpha_{3} \\
\alpha_{3} & \alpha_{4} & -\alpha_{1} & -\alpha_{2} \\
\alpha_{4} & -\alpha_{3} & \alpha_{2} & -\alpha_{1}
\end{array}\right] .
$$

For a given ROD, the calculation of its CROD is given in [13].

For the CRODs as defined in equations (7)-(9), it can be easily shown that the following equality

$$
\mathcal{G}_{c}\left[\mathcal{G}_{c}\right]^{T}=\sum_{j=1}^{m} \alpha_{j}^{2} \cdot \mathbf{I}
$$

holds true, where the superscript ${ }^{T}$ stands for the transpose (without conjugate!) of a matrix or vector. As can be seen from the discussion in Section "Simulation Results", one can remove the inter-symbol interference (ISI) by using the above property of CROD, but the diversity gain thus obtained from the multiple channels is limited when the channel is complex instead of real.

To find the decoding scheme, let us consider the property of $\mathcal{G}_{c}\left[\mathcal{G}_{c}\right]^{H}$, where the superscript ${ }^{H}$ stands for the conjugate transpose of a matrix or vector. We have

$$
\mathcal{G}_{c}^{(2)}\left[\mathcal{G}_{c}^{(2)}\right]^{H}=\left[\begin{array}{cc}
\sum_{i=1}^{2}\left|\alpha_{i}\right|^{2} & \alpha_{1} \alpha_{2}^{H}-\alpha_{1}^{H} \alpha_{2} \\
\star & \sum_{i=1}^{2}\left|\alpha_{i}\right|^{2}
\end{array}\right],
$$

$\mathcal{G}_{c}^{(3)}\left[\mathcal{G}_{c}^{(3)}\right]^{H}=\left[\begin{array}{cccc}\sum_{i=1}^{3}\left|\alpha_{i}\right|^{2} & \alpha_{1} \alpha_{2}^{H}-\alpha_{1}^{H} \alpha_{2} & \alpha_{1} \alpha_{3}^{H}-\alpha_{1}^{H} \alpha_{3} & -\alpha_{2} \alpha_{3}^{H}+\alpha_{2}^{H} \alpha_{3} \\ \star & \sum_{i=1}^{3}\left|\alpha_{i}\right|^{2} & \alpha_{2} \alpha_{3}^{H}-\alpha_{2}^{H} \alpha_{3} & \alpha_{1} \alpha_{3}^{H}-\alpha_{1}^{H} \alpha_{3} \\ \star & \star & \sum_{i=1}^{3}\left|\alpha_{i}\right|^{2} & -\alpha_{1} \alpha_{2}^{H}+\alpha_{1}^{H} \alpha_{2} \\ \star & \star & \star & \sum_{i=1}^{3}\left|\alpha_{i}\right|^{2}\end{array}\right]$, where the entry marked with $\star$ means that its value can be inferred from the value of its corresponding symmetric entry. It can be checked that the structural property as shown in equations (11)-(13) also holds true for higher dimensional CRODs.

Using RODs and the corresponding CRODs, a general space-time encoding scheme and two decoding approaches for RFID-MIMO systems can be developed as follows.

Consider the equivalent RFID-MIMO channel (3). Denote by $T_{f}$ a symbol period. Suppose that the channels of both forward and backward links do not change with time during a coding block period $K T_{f}$. The transmit signal $\mathbf{x}$ at the reader is also fixed during one coding block period $K T_{f}$. Therefore, the equivalent composite channel $\mathbf{H}^{\mathrm{b}} \breve{\mathbf{H}}$ will not change with time when we only consider the signal processing for one coding block. Let us define

$$
\mathbf{A}=\mathbf{H}^{\mathrm{b}} \breve{\mathbf{H}} .
$$

Let $\mathcal{G}$ (of dimension $N_{\text {tag }} \times K$ ) be a ROD in variables $\mathcal{S}_{1}, \mathcal{S}_{2}, \ldots, \mathcal{S}_{K}$, where $\mathcal{S}_{1}, \mathcal{S}_{2}, \ldots, \mathcal{S}_{K}$ are the symbols to be transmitted at the $N_{\text {tag }}$ transmit antennas in one STC frame. Define

$$
\mathbf{w}(t)=\left[\begin{array}{c}
w(t) \\
w\left(t-T_{f}\right) \\
\vdots \\
w\left(t-(K-1) T_{f}\right)
\end{array}\right],
$$

where $w(t)$ is the baseband waveform of the transmit signal at the tag. The transmitted signal across the $N_{\text {tag }}$ transmit antennas at the tag can be expressed as

$$
\boldsymbol{\gamma}(t)=\sqrt{\frac{E_{0}}{N_{\mathrm{tag}}}} \mathcal{G}\left(\mathcal{S}_{1}, \mathcal{S}_{2}, \ldots, \mathcal{S}_{K}\right) \mathbf{w}(t),
$$

where $E_{0}$ is the total power used for the transmission of one symbol per time slot. The scaling coefficient $\sqrt{\frac{E_{0}}{N_{\text {tag }}}}$ is to normalize the overall energy consumption per time slot

$$
\begin{aligned}
& \mathcal{G}_{c}^{(4)}\left[\mathcal{G}_{c}^{(4)}\right]^{H}= \\
& {\left[\begin{array}{cccc}
\sum_{i=1}^{4}\left|\alpha_{i}\right|^{2} & \alpha_{1} \alpha_{2}^{H}-\alpha_{1}^{H} \alpha_{2}-\alpha_{3} \alpha_{4}^{H}+\alpha_{3}^{H} \alpha_{4} & \alpha_{1} \alpha_{3}^{H}-\alpha_{1}^{H} \alpha_{3}+\alpha_{2} \alpha_{4}^{H}-\alpha_{2}^{H} \alpha_{4} & \alpha_{1} \alpha_{4}^{H}-\alpha_{1}^{H} \alpha_{4}-\alpha_{2} \alpha_{3}^{H}+\alpha_{2}^{H} \alpha_{3} \\
\star & \sum_{i=1}^{4}\left|\alpha_{i}\right|^{2} & \alpha_{2} \alpha_{3}^{H}-\alpha_{2}^{H} \alpha_{3}-\alpha_{1} \alpha_{4}^{H}+\alpha_{1}^{H} \alpha_{4} & \alpha_{2} \alpha_{4}^{H}-\alpha_{2}^{H} \alpha_{4}+\alpha_{1} \alpha_{3}^{H}-\alpha_{1}^{H} \alpha_{3} \\
\star & \star & \sum_{i=1}^{4}\left|\alpha_{i}\right|^{2} & \alpha_{3} \alpha_{4}^{H}-\alpha_{3}^{H} \alpha_{4}-\alpha_{1} \alpha_{2}^{H}+\alpha_{1}^{H} \alpha_{2} \\
\star & \star & \star & \sum_{i=1}^{4}\left|\alpha_{i}\right|^{2}
\end{array}\right],}
\end{aligned}
$$


at the tag side to be $E_{0}$ no matter how many antennas are deployed at the tag.

\section{Two Space-Time Decoding Approaches for RFID MIMO Systems}

The received signal after sampling can be expressed as

$$
\overrightarrow{\mathbf{y}}=\sqrt{\frac{E_{0}}{N_{\operatorname{tag}}}} \mathbf{A} \mathcal{G}\left(\mathcal{S}_{1}, \mathcal{S}_{2}, \ldots, \mathcal{S}_{K}\right)+\overrightarrow{\mathbf{n}},
$$

where $\overrightarrow{\mathbf{n}}$ is the receiver noise (a matrix) at the corresponding time instant. Notice that $\overrightarrow{\mathbf{y}}$ is of dimension $N_{\text {rd }} \times K$, since one frame of the transmitted signal contains the pulses of $K$ time slots.

Denote by $[\mathbf{M}]_{j}$ the $j$ th row of a matrix $\mathbf{M}$. Let us consider the $j$ th row of the matrix $\overrightarrow{\mathbf{y}}$ which is the received signal at the $j$ th antenna of the reader for the time instants $1, \ldots, K$ respectively. Let

$$
[\mathbf{A}]_{j}=\left[\begin{array}{llll}
\alpha_{j 1} & \alpha_{j 2} & \cdots & \alpha_{j N_{\text {tag }}}
\end{array}\right] .
$$

Since the transmitted signal is space-time coded, the entries in $[\mathbf{y}]_{j}$ should be related with each other somehow. Right-hand multiplying both sides of equation (15) with the matrix $\left[\mathcal{G}_{c}\left(\alpha_{j 1}, \alpha_{j 2}, \ldots, \alpha_{j N_{\mathrm{tag}}}\right)\right]^{T}$, we have

$$
\begin{aligned}
& \mathbf{z}_{j}:=[\overrightarrow{\mathbf{y}}]_{j}\left[\mathcal{G}_{c}\left(\alpha_{j 1}, \alpha_{j 2}, \ldots, \alpha_{j N_{\mathrm{tag}}}\right)\right]^{T} \\
& =\left\{\sqrt{\frac{E_{0}}{N_{\text {tag }}}}[\mathbf{A}]_{j} \mathcal{G}\left(\mathcal{S}_{1}, \mathcal{S}_{2}, \ldots, \mathcal{S}_{K}\right)+[\mathbf{n}]_{j}\right\} \\
& \times\left[\mathcal{G}_{c}\left(\alpha_{j 1}, \alpha_{j 2}, \ldots, \alpha_{j N_{\mathrm{tag}}}\right)\right]^{T} \\
& =\left\{\sqrt{\frac{E_{0}}{N_{\operatorname{tag}}}}\left[\mathcal{S}_{1} \mathcal{S}_{2} \ldots \mathcal{S}_{K}\right] \mathcal{G}_{c}\left(\alpha_{j 1}, \alpha_{j 2}, \ldots, \alpha_{j N_{\mathrm{tag}}}\right)+[\overrightarrow{\mathbf{n}}]_{j}\right\} \\
& \times\left[\mathcal{G}_{c}\left(\alpha_{j 1}, \alpha_{j 2}, \ldots, \alpha_{j N_{\mathrm{tag}}}\right)\right]^{T} \\
& =\sqrt{\frac{E_{0}}{N_{\text {tag }}}} \sum_{k=1}^{N_{\text {tag }}}\left[\alpha_{j k}\right]^{2}\left[\begin{array}{llll}
\mathcal{S}_{1} & \mathcal{S}_{2} & \cdots & \mathcal{S}_{K}
\end{array}\right]+[\overrightarrow{\mathbf{n}}]_{j} \\
& \times\left[\mathcal{G}_{c}\left(\alpha_{j 1}, \alpha_{j 2}, \ldots, \alpha_{j N_{\text {tag }}}\right)\right]^{T} .
\end{aligned}
$$

From equation (17) we can see that the transmitted symbols $\mathcal{S}_{1}, \mathcal{S}_{2}, \ldots, \mathcal{S}_{K}$ are decoupled from each other in the processed signal $\mathbf{z}_{j}$ through the processing algorithm (16). However, it is not efficient to decode the symbols $\mathcal{S}_{1}, \mathcal{S}_{2}, \ldots, \mathcal{S}_{K}$ directly from (17) since the complex channel makes the phase of $\sum_{k=1}^{N_{\text {tag }}}\left[\alpha_{j k}\right]^{2}$ randomly change over $[0,2 \pi]$. Define

$$
\beta_{j}=\sum_{k=1}^{N_{\mathrm{tag}}}\left[\alpha_{j k}\right]^{2} .
$$

Multiplying both sides of (17) by $\beta_{j}^{H}$ will remove the phase ambiguity of the equivalent channel. This gives

$$
\overline{\mathbf{z}}_{j}:=\beta_{j}^{H} \mathbf{z}_{j}=\left|\beta_{j}\right|^{2}\left[\begin{array}{llll}
\mathcal{S}_{1} & \mathcal{S}_{2} & \cdots & \mathcal{S}_{K}
\end{array}\right]+\overline{\mathbf{n}}_{j},
$$

where

$$
\overline{\mathbf{n}}_{j}=\beta_{j}^{H}[\overrightarrow{\mathbf{n}}]_{j}\left[\mathcal{G}_{c}\left(\alpha_{j 1}, \alpha_{j 2}, \ldots, \alpha_{j N_{\text {tag }}}\right)\right]^{T} .
$$

To collect all the diversities provided by multiple receive antennas at the reader, we sum up all $\overline{\mathbf{z}}_{j}$ 's. This gives

$$
\overline{\mathbf{z}}:=\sum_{j=1}^{N_{\mathrm{rd}}} \overline{\mathbf{z}}_{j}=\sum_{j=1}^{N_{\mathrm{rd}}}\left|\beta_{j}\right|^{2}\left[\begin{array}{llll}
\mathcal{S}_{1} & \mathcal{S}_{2} & \cdots & \mathcal{S}_{K}
\end{array}\right]+\sum_{j=1}^{N_{\mathrm{rd}}} \overline{\mathbf{n}}_{j} .
$$

The symbols $\mathcal{S}_{1}, \mathcal{S}_{2}, \ldots, \mathcal{S}_{K}$ can be easily decoded from equation (20).

For the convenience of exposition in next section, we call the encoding and decoding scheme discussed above as Scheme I.

Another decoding scheme (hereafter it is referred to as Scheme II) is to exploit the property of the matrix $\mathcal{G}_{c}\left(\mathcal{G}_{c}\right)^{H}$, as shown in equations (11)-(13). Right-hand multiplying both sides of equation (15) with the matrix $\left[\mathcal{G}_{c}\left(\alpha_{j 1}, \alpha_{j 2}, \ldots, \alpha_{j N_{\text {tag }}}\right)\right]^{H}$, we have

$$
\begin{aligned}
\mathbf{u}_{j}:= & {[\overrightarrow{\mathbf{y}}]_{j}\left[\mathcal{G}_{c}\left(\alpha_{j 1}, \alpha_{j 2}, \ldots, \alpha_{j N_{\mathrm{tag}}}\right)\right]^{H} } \\
= & \left\{\sqrt{\frac{E_{0}}{N_{\mathrm{tag}}}}\left[\mathcal{S}_{1} \mathcal{S}_{2} \ldots \mathcal{S}_{K}\right] \mathcal{G}_{c}\left(\alpha_{j 1}, \alpha_{j 2}, \ldots, \alpha_{j N_{\mathrm{tag}}}\right)+[\overrightarrow{\mathbf{n}}]_{j}\right\} \\
& \times\left[\mathcal{G}_{c}\left(\alpha_{j 1}, \alpha_{j 2}, \ldots, \alpha_{j N_{\mathrm{tag}}}\right)\right]^{H} \\
= & \sqrt{\frac{E_{0}}{N_{\mathrm{tag}}}}\left[\mathcal{S}_{1} \mathcal{S}_{2} \ldots \mathcal{S}_{K}\right] \cdot\left[\mathcal{G}_{c}\left(\alpha_{j 1}, \alpha_{j 2}, \ldots, \alpha_{j N_{\mathrm{tag}}}\right)\right] \\
& \times\left[\mathcal{G}_{c}\left(\alpha_{j 1}, \alpha_{j 2}, \ldots, \alpha_{j N_{\mathrm{tag}}}\right)\right]^{H}+[\overrightarrow{\mathbf{n}}]_{j} \\
& \times\left[\mathcal{G}_{c}\left(\alpha_{j 1}, \alpha_{j 2}, \ldots, \alpha_{j N_{\mathrm{tag}}}\right)\right]^{H} .
\end{aligned}
$$

From equations (21) and (11)-(13) we can see that, if the symbols $\mathcal{S}_{1}, \mathcal{S}_{2}, \ldots, \mathcal{S}_{K}$ are real, the symbol to be decoded, say $\mathcal{S}_{k}$ for some $k$, and the ISI caused by other symbols, are projected into different subspaces in the complex plane: the desired signal is in the real subspace, while the ISI is in the imaginary subspace. Therefore, a very simple decoding method for this case works in the following way: From $k$ th entry of $\mathbf{u}_{j}$ (denoted as $\mathbf{u}_{j, k}$ ), get the real part of $\mathbf{u}_{j, k}$ [denoted as $\operatorname{Re}\left(\mathbf{u}_{j, k}\right)$ ], and then decode $\mathcal{S}_{k}$ in terms of $\operatorname{Re}\left(\mathbf{u}_{j, k}\right)$.

The diversities provided by multiple receive antennas at the reader can be collected in the following way:

$$
\overline{\mathbf{u}}_{k}:=\sum_{j=1}^{N_{\mathrm{rd}}} \operatorname{Re}\left(\mathbf{u}_{j, k}\right) .
$$

Then $\mathcal{S}_{k}$ can be decoded in terms of $\overline{\mathbf{u}}_{k}$.

\section{Simulation Results}

In this section, we investigate the symbol-error rate (SER) or bit-error rate (BER) performance of both Schemes I 
and II. In Scheme I, the quadrature phase shift keying (QPSK) modulation is used and the constellation of transmitted symbols is $\frac{ \pm 1 \pm j}{\sqrt{2}}$. In Scheme II, the binary phase shift keying (BPSK) modulation is used and the constellation of transmitted symbols is \pm 1 . Therefore, the SER in Scheme II reduces to BER. At the transmitter of the reader, the signal $\mathbf{x}$ takes the form of a random vector whose entry is uniformly distributed among $\pm \frac{1}{\sqrt{N_{\text {rd }}}}$. It is seen that $\mathbf{x}$ is of unity power. Each entry of the channels $\mathbf{H}^{\mathrm{f}}$ and $\mathbf{H}^{\mathrm{b}}$ is of mean zero and variance unity.

In the figures to be shown, the signal-to-noise power ratio (SNR) is defined as the $\frac{E_{0}}{\sigma_{\mathbf{n}}^{2}}$, where $\sigma_{\overrightarrow{\mathbf{n}}}^{2}$ is the variance of the each entry of noise vector $\overrightarrow{\mathbf{n}}$.

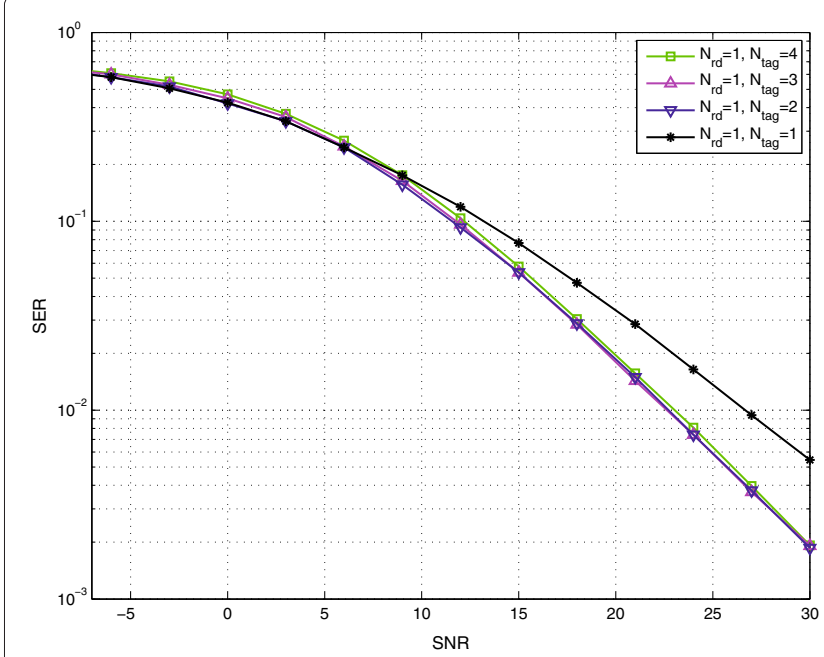

(a) SER vs $\mathrm{N}_{\text {tag }}$ for $\mathrm{N}_{\mathrm{rd}}=1$

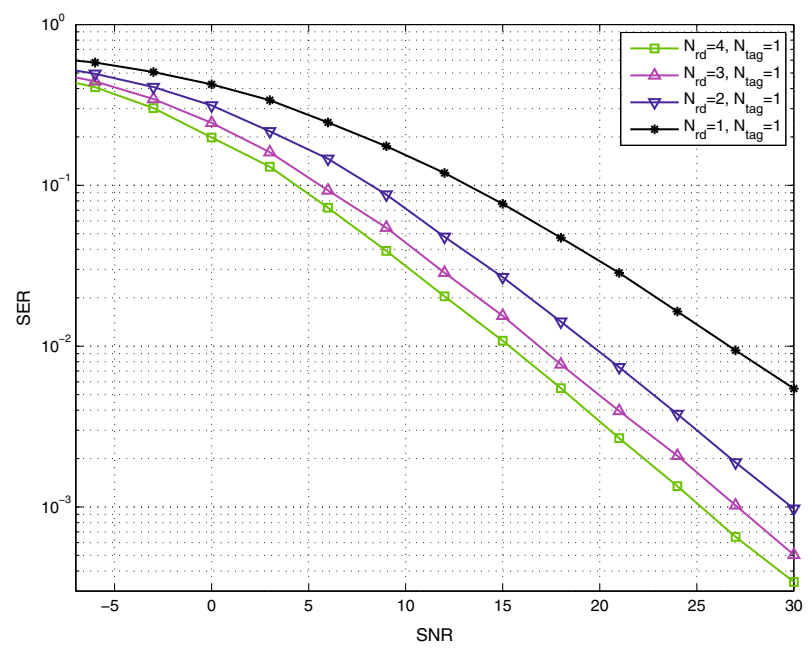

(c) SER vs $\mathrm{N}_{\mathrm{rd}}$ for $\mathrm{N}_{\mathrm{tag}}=1$
Figure 2 shows the SER of Scheme I for different cases: Figures 2(a) and (b) illustrate how the SER changes with $N_{\text {tag }}$ for fixed $N_{\text {rd }}$, i.e., when $N_{\text {rd }}=1$ and 4 respectively; while Figures 2(c) and (d) demonstrate how the SER changes with $N_{\text {rd }}$ for fixed $N_{\text {tag }}$, i.e., when $N_{\text {tag }}=1$ and 4 respectively.

Figure 3 shows the BER of Scheme II for different cases: Figures 3(a) and (b) illustrate how the BER changes with $N_{\text {tag }}$ for fixed $N_{\text {rd }}$, i.e., when $N_{\text {rd }}=1$ and 4 respectively; while Figures 3(c) and (d) demonstrate how the BER changes with $N_{\text {rd }}$ for fixed $N_{\text {tag }}$, i.e., when $N_{\text {tag }}=1$ and 4 respectively.

From Figures 2 and 3 the following phenomena can be observed:

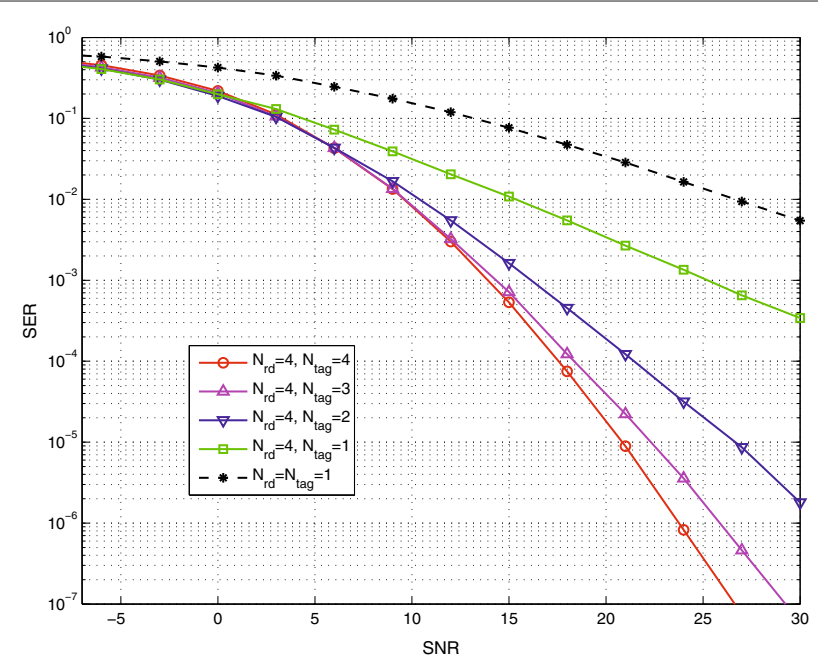

(b) SER vs $\mathrm{N}_{\text {tag }}$ for $\mathrm{N}_{\mathrm{rd}}=4$

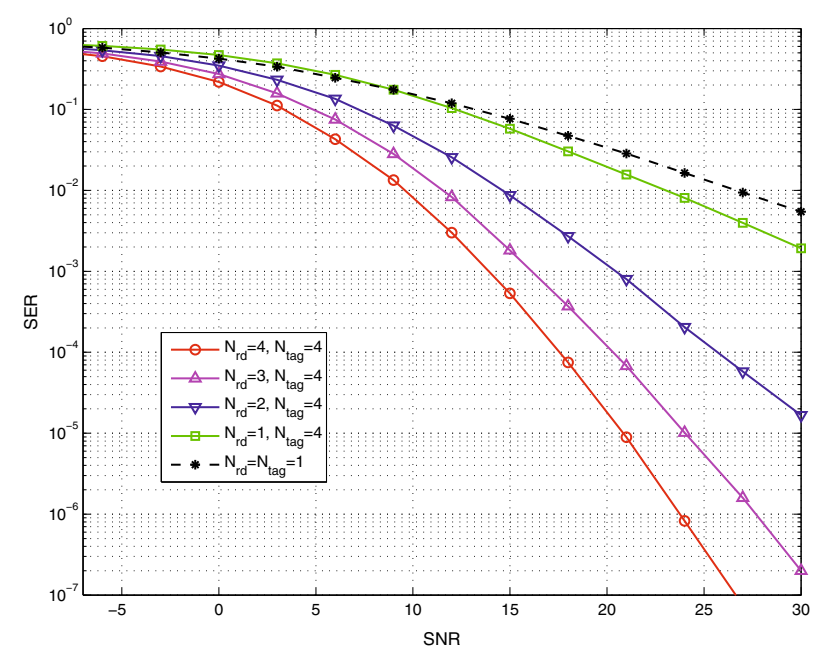

(d) SER vs $\mathrm{N}_{\mathrm{rd}}$ for $\mathrm{N}_{\text {tag }}=4$

Figure 2 SER of RFID MIMO systems for Scheme I with QPSK modulation. (a)SER vS $N_{\text {tag }}$ for $N_{\text {rd }}=1$ (b)SER vs $N_{\text {tag }}$ for $N_{\text {rd }}=4$ (c)SER vs $N_{\text {rd }}$ for $N_{\text {tag }}=1$ (d)SER vs $N_{\text {rd }}$ for $N_{\text {tag }}=4$. 


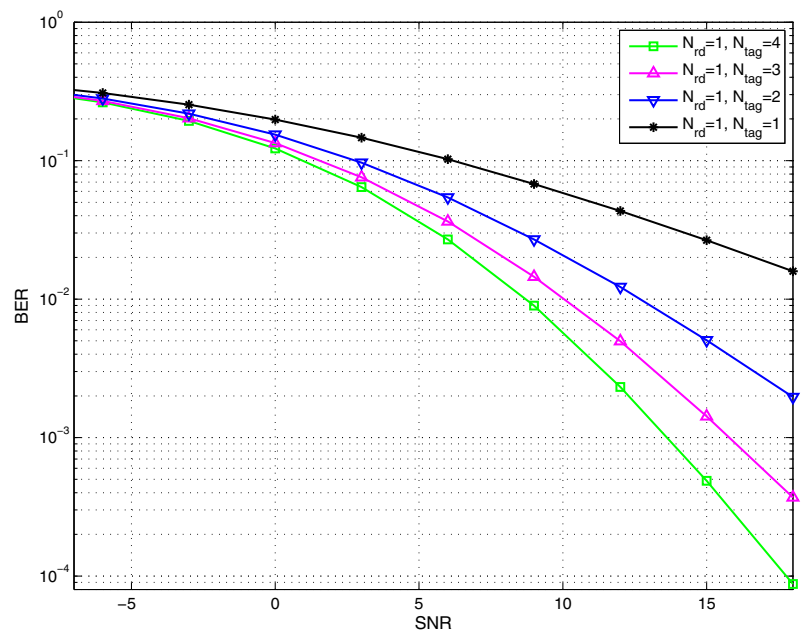

(a) BER vs $\mathrm{N}_{\text {tag }}$ for $\mathrm{N}_{\mathrm{rd}}=1$

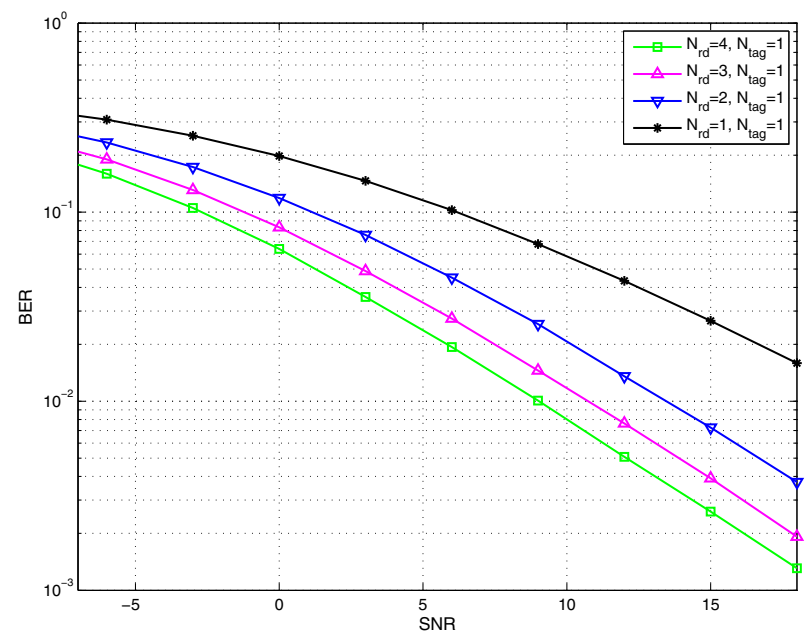

(c) BER vs $\mathrm{N}_{\mathrm{rd}}$ for $\mathrm{N}_{\mathrm{tag}}=1$

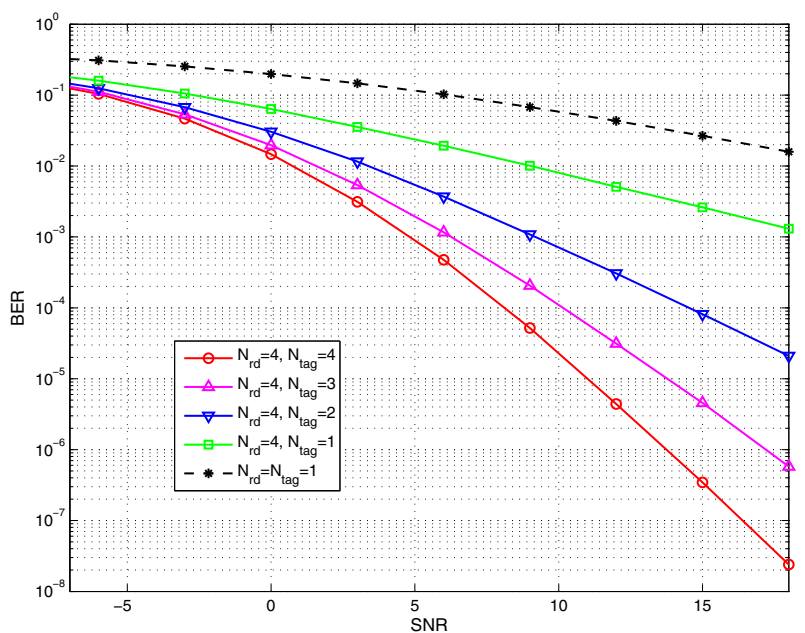

(b) BER vs $\mathrm{N}_{\text {tag }}$ for $\mathrm{N}_{\mathrm{rd}}=4$

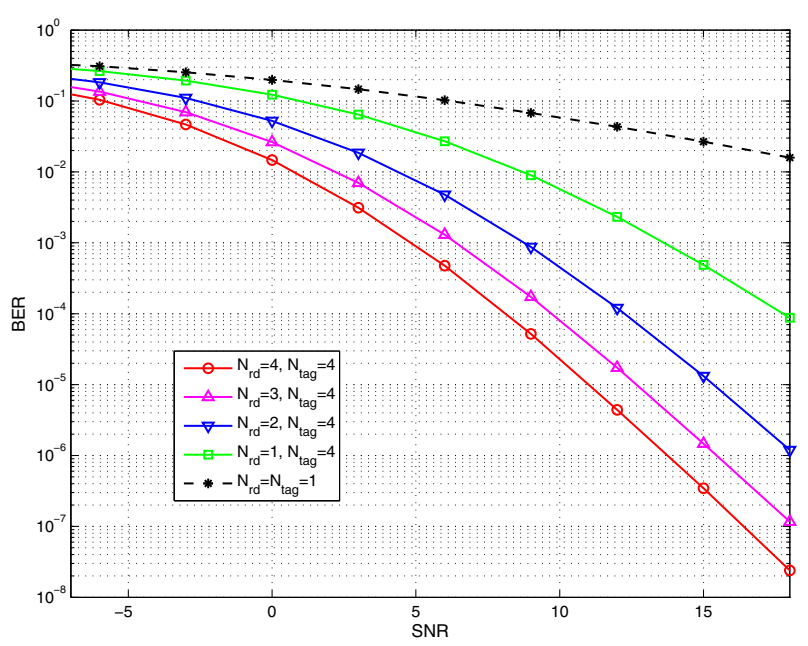

(d) BER vs $\mathrm{N}_{\mathrm{rd}}$ for $\mathrm{N}_{\text {tag }}=4$

Figure 3 BER of RFID MIMO systems for Scheme II with BPSK modulation. (a)BER vs $N_{\text {tag }}$ for $N_{\text {rd }}=1$ (b)BER vs $N_{\text {tag }}$ for $N_{\text {rd }}=4$ (c)BER vs $N_{\text {rd }}$ for $N_{\text {tag }}=1$ (d)BER vs $N_{\text {rd }}$ for $N_{\text {tag }}=4$.

Claim 1. Comparing the dashed curves, which corresponds to the performance of the non space-time encoded RFID system with single antenna at both reader and tag sides, and the solid curves in Figures 2(b), (d), and Figures 3(b), (d), we see that deploying multiple antennas at both reader and tag can greatly improve the SER/BER performance of RFID systems.

Claim 2. When $N_{\text {rd }}$ is fixed to be one, increasing $N_{\text {tag }}$ considerably decreases the BER of the system in Scheme II, but only marginally decreases the SER of the system in Scheme I. For example, when $\mathrm{SNR}=18 \mathrm{~dB}$ and $N_{\mathrm{rd}}=1$, the BER of Scheme II decreases from $1.6 \times 10^{-2}$ at $N_{\text {tag }}=1$ to $2.0 \times 10^{-3}$ at $N_{\text {tag }}=2$ and $8.8 \times 10^{-5}$ at $N_{\text {tag }}=4$, respectively. For the same SNR and $N_{\text {rd }}$, the SER of Scheme I decreases from $4.7 \times 10^{-2}$ at $N_{\text {tag }}=1$ to $2.9 \times 10^{-2}$ at $N_{\text {tag }}=2$ and $3.0 \times 10^{-2}$ at $N_{\text {tag }}=4$ respectively. The reason for this phenomenon is that the channel diversity provided by $N_{\text {tag }}$ antennas at the tag side is harvested by Scheme II [as seen from equations (11)-(13)], but not harvested by Scheme I [as seen from equation (17)].

Claim 3. When $N_{\text {tag }}$ is fixed to be one, increasing $N_{\text {rd }}$ noticeably and monotonically decreases the SER or BER of the system. This phenomenon can be clearly seen from Figure 2(c) and Figure 3(c). The reason is that only the array gain is provided by the system when $N_{\text {tag }}=1$ and it is indeed collected by both Scheme I and Scheme II. Due to the double Rayleigh fading channel, the system performance cannot be improved conspicuously by only exploiting this array gain. 
Claim 4. When $N_{\text {rd }}$ (or $N_{\text {tag }}$ ) is fixed and greater than one, increasing $N_{\text {tag }}$ (or $N_{\text {rd }}$ ) greatly decreases the SER or BER of the system, especially for Scheme II. For example, when $\mathrm{SNR}=18 \mathrm{~dB}$ and $N_{\mathrm{tag}}=4$, the SER of Scheme I decreases from $3.0 \times 10^{-2}$ at $N_{\text {rd }}=1$ to $2.7 \times 10^{-3}$ at $N_{\text {rd }}=2$ and $7.5 \times 10^{-5}$ at $N_{\text {rd }}=4$, respectively. For the same SNR and $N_{\text {tag }}$, the BER of Scheme II decreases from $8.8 \times 10^{-5}$ at $N_{\text {rd }}=1$ to $1.2 \times 10^{-6}$ at $N_{\text {rd }}=2$ and $2.4 \times 10^{-8}$ at $N_{\text {tag }}=4$ respectively. To achieve the $\mathrm{BER}=8.8 \times 10^{-5}$ for the case of Scheme II and $N_{\text {tag }}=4$, the SNR gain is about $7.5 \mathrm{~dB}$ and $10 \mathrm{~dB}$, respectively, by deploying $N_{\text {rd }}=2$ and $N_{\text {rd }}=4$ antennas at the reader, compared to the single-antenna setup at the reader. On the other side, to achieve the $\mathrm{BER}=1.3 \times 10^{-3}$ for the case of Scheme II and $N_{\text {rd }}=4$, the SNR gain is about $9 \mathrm{~dB}$ and $13.5 \mathrm{~dB}$, respectively, by deploying $N_{\mathrm{tag}}=2$ and $N_{\mathrm{tag}}=4$

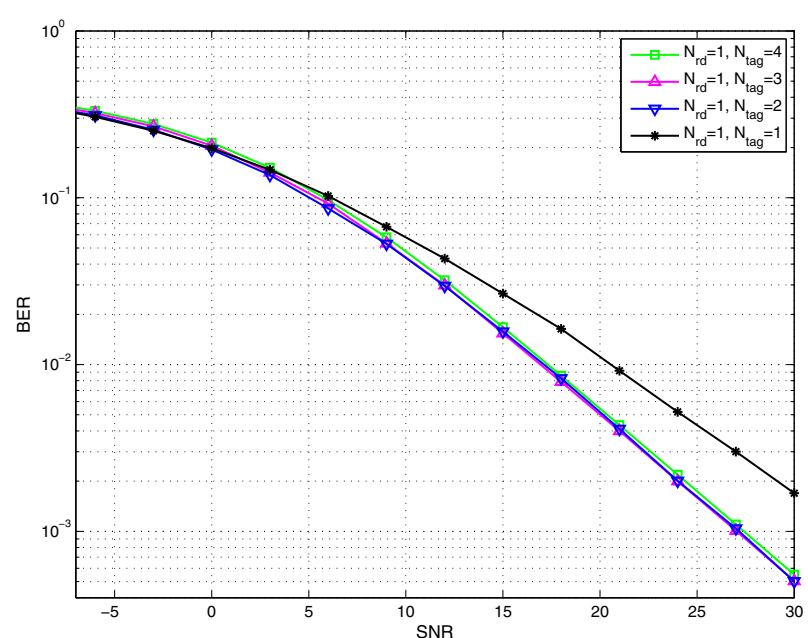

(a) BER vs $\mathrm{N}_{\text {tag }}$ for $\mathrm{N}_{\mathrm{rd}}=1$

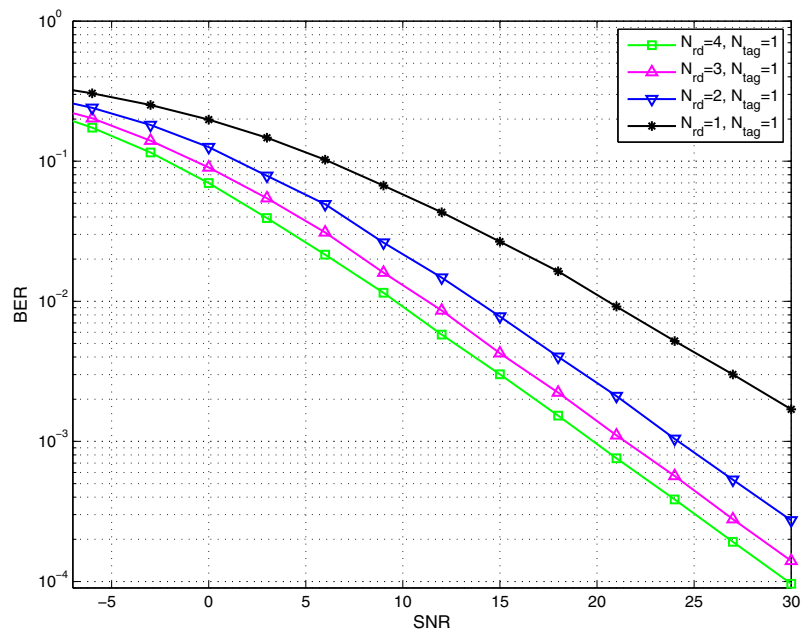

(c) BER vs $\mathrm{N}_{\mathrm{rd}}$ for $\mathrm{N}_{\mathrm{tag}}=1$ antennas at the tag, compared to the single-antenna setup at the tag. This is dramatic improvement for the system performance.

Claim 5. Scheme II yields much better SER performance than Scheme I. There are two reasons. The first reason, which is obvious, is that different symbol constellations are used in Schemes I and II. In the above simulations, one symbol in Scheme I actually carries two bit information, while one symbol in Scheme II carries only one bit information. The second reason, which is somewhat subtle to see, is that the diversity gain harvested by Scheme I is lower than that harvested by Scheme II, even though Scheme II throw away the signal in another half signal space. This observation can be seen by comparing equations (11)-(13) and (22) (for Scheme II) and equations

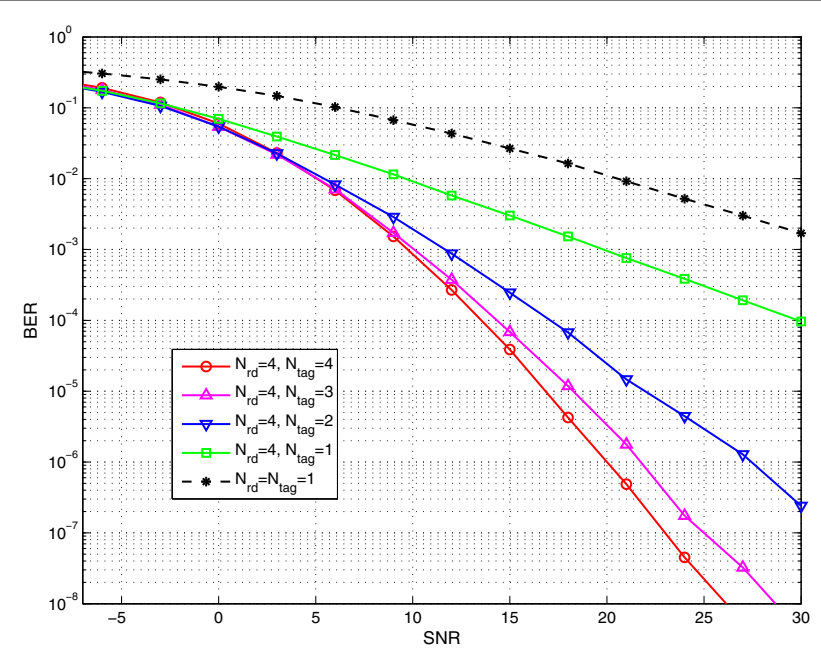

(b) BER vs $\mathrm{N}_{\text {tag }}$ for $\mathrm{N}_{\mathrm{rd}}=4$

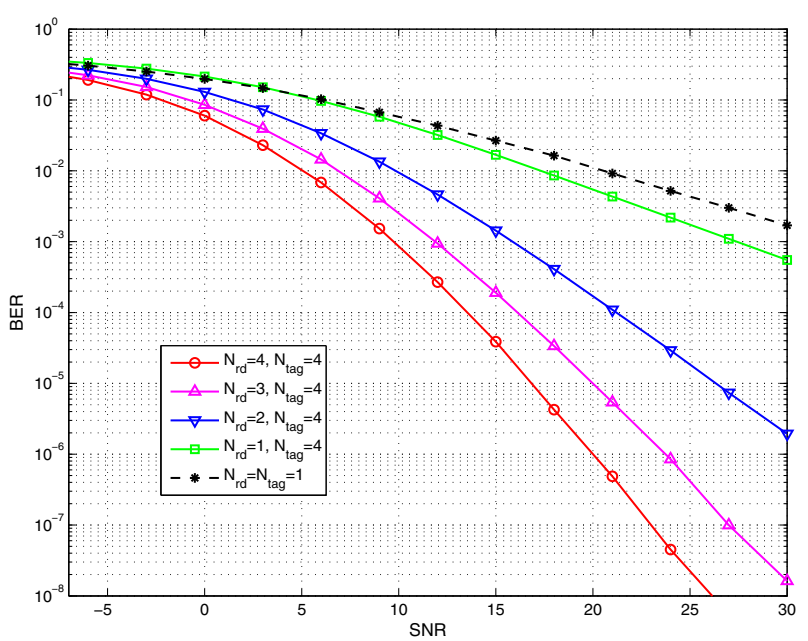

(d) BER vs $\mathrm{N}_{\mathrm{rd}}$ for $\mathrm{N}_{\operatorname{tag}}=4$

Figure 4 BER of RFID MIMO systems for Scheme I with BPSK modulation. (a)BER vs $N_{\text {tag }}$ for $N_{\text {rd }}=1$ (b)BER vs $N_{\text {tag }}$ for $N_{\text {rd }}=4$ (c)BER vs $N_{\text {rd }}$ for $N_{\text {tag }}=1$ (d)BER vs $N_{\text {rd }}$ for $N_{\text {tag }}=4$. 
(17), (18) and (20) (for Scheme I). For Scheme I, it is seen from (17) and (18) that the $N_{\text {tag }}$ independent channels are not coherently summed. In (20), the $N_{\text {rd }}$ independent summed-channels are further summed. Thus Scheme I yields a diversity order of $N_{\text {rd }}$ and the system-inherited diversity order $N_{\text {tag }}$ is sacrificed. For Scheme II, it is seen from (11)-(13) that the $N_{\text {tag }}$ independent channels are first coherently summed, yielding a diversity order of $N_{\text {tag }}$. From (22), the $N_{\text {rd }}$ independent summed-channels are further summed, yielding a diversity order of $N_{\text {rd }}$. Thus a total diversity order of $N_{\text {rd }} \times N_{\text {tag }}$ is obtained in Scheme II.

Claim 6. Comparing Figure 2 and Figure 3, we can conclude that it is better to deploy as many antennas as possible at the reader. At least the number of antennas at the reader side should be not less than the number of antennas at the tag side. In this way, the full channel diversity generated by multiple antennas at the tag can be maximally exploited.

It may be argued that it is not fair to compare the SER performance of Scheme I and Scheme II, since the former uses QPSK modulation, while the latter uses BPSK modulation. To make the comparison complete, the BER performance of Scheme I with BPSK modulation is shown in Figure 4 for the corresponding cases. Figure 2, Figure 4 and Figure 3 show that the BER performance of Scheme I is much worse than that of Scheme II, even though the BER of Scheme I with BPSK modulation is lower than the SER of Scheme I with QPSK modulation for the same configuration of antenna numbers at the reader and tag. By comparing Figure 4 and Figure 3 we can see that Claims 1-6 obtained based on the comparison between Figure 2 and Figure 3 also holds true qualitatively.

From the above phenomena, the following conclusions can be drawn: if the required data rate is not high, it is better to use real-symbol constellation for the transmitted symbols at the tag and correspondingly to use Scheme II decoding policy at the reader receiver; by keeping the cost of the system under constraint, it is better to deploy multiple tag antennas and reader antennas, and the number of reader antennas should be at least equal to the number of tag antennas.

It is interesting to compare the ROD based STC and Alamouti STC. Figure 5 shows the comparison. It can be seen that Scheme II and Alamouti STC yield the same BER performance, both are better than Scheme I. This is due to the fact that both Scheme II and Alamouti STC collect all the available channel diversities, while Scheme I does not.

Finally, let us compare the complexity of Scheme I and Scheme II. Both Scheme I and Scheme II perform the same processing, as shown in equations (4)-(6), for the transmitted symbols at the tag. As seen from (4)-(6), the symbol processing at the tag is quite simple: only the sign of the symbols to be transmitted needs to be changed at some time slots for some antennas. For the processing of a block of space-time decoding at the reader, Scheme I needs $N_{\mathrm{rd}}\left(K^{2}+K+N_{\mathrm{tag}}\right)$ complex multiplications and $N_{\text {rd }} K(K-1)+\left(N_{\text {rd }}-1\right) K+N_{\text {rd }}\left(N_{\text {tag }}-1\right)=N_{\text {rd }}\left(K^{2}+\right.$ $\left.N_{\text {tag }}-1\right)-K$ complex additions, and Scheme II needs

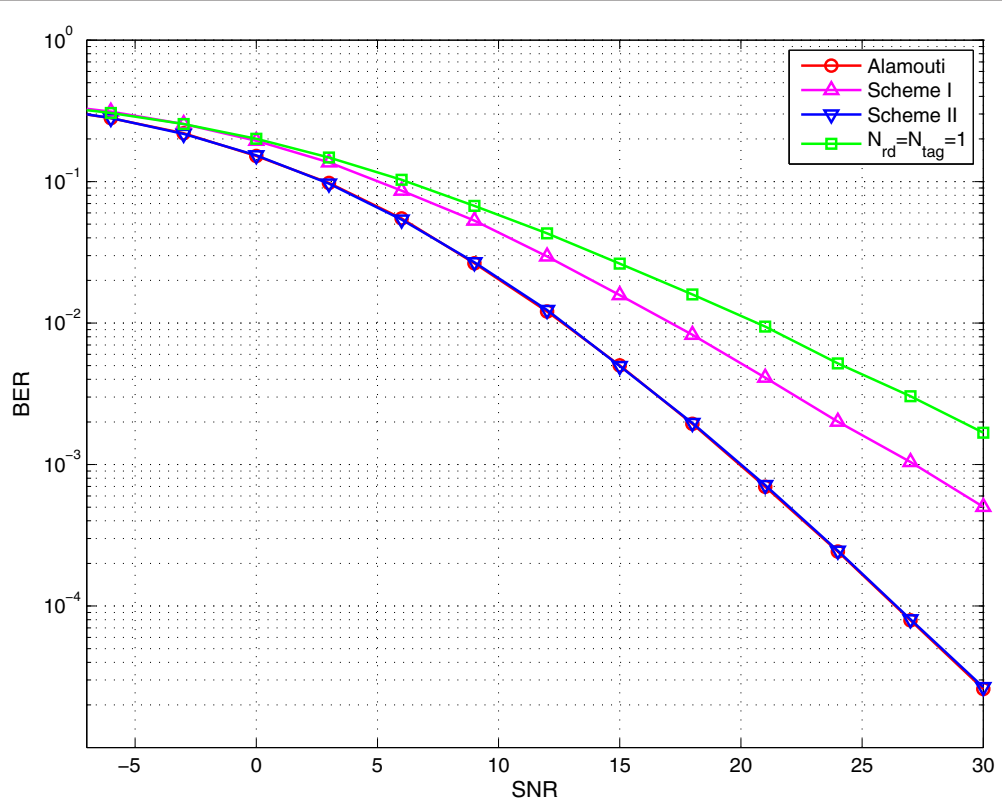

Figure 5 A comparison among Scheme I, Scheme II and the Alamouti STC. For the curves marked with "Scheme I", "Scheme II" and "Alamouti", $N_{\text {tag }}=2$ and $N_{\text {rd }}=1$. 
$N_{\text {rd }} K^{2}$ complex multiplications, $N_{\text {rd }} K(K-1)$ complex additions, and $\left(N_{\mathrm{rd}}-1\right) K$ real additions. Therefore, the computational burden of Scheme II is a little less than that of Scheme I. With regard to the hardware cost of the proposed STC technique, the main increase in the cost arises from the deployment of multiple antennas. The cost increase for the involved signal processing unit is negligible at either tags or readers, since the space-time encoding is very simple, which can be easily dealt with by the embedded chip at tags, and the required computational burden for the space-time decoding at readers is also negligible compared to the relatively strong computation power of readers.

\section{Conclusions}

In this paper, we have discussed the space-time encoding and decoding problem for RFID MIMO systems. First, a mathematical model for this kind of system is developed from the viewpoint of signal processing, which makes it easy to design the STC schemes. Two STC schemes, namely Scheme I and Scheme II, are proposed. Simulation results illustrate that the proposed approaches can greatly improve the SER/BER performance of RFID systems, compared to non space-time encoded RFID systems. Besides, the SER/BER performance for Scheme I and Scheme II is thoroughly compared and it is found that Scheme II with the innate real-symbol constellation yields better SER/BER performance than Scheme I.

As is commonly assumed in the STC technique, the channel state information (CSI) is required to be available at the receiver side of the reader to adopt the technology of Scheme I and Scheme II. The channel estimation problem for RFID systems has been discussed in $[8,10]$, where a method for estimating the channel of the whole chain, including forward link, backscattering coefficient, and backward link, is presented. However, to estimate the forward and backford channels $\mathbf{H}^{\mathrm{f}}$ and $\mathbf{H}^{\mathrm{b}}$ separately remains an open issue. On the other hand, if the CSI is also available at the transmitter side of the reader, we can combine the design for the reader transmit signal and STC for the tag to further improve the system performance. For the first step towards the optimal transmit signal design at the reader side, readers are referred to the reference [14].

\section{Competing interests}

The authors declare that they have no competing interests.

Received: 15 June 2012 Accepted: 16 August 2012

Published: 29 October 2012

\section{References}

1. JD Griffin, GD Durgin, Gains for RF tags using multiple antennas. IEEE Trans. Antennas Propag. 56, 563-570 (2008)

2. NC Karmakar (ed.), Handbook of Smart Antennas for RFID Systems. (Wiley, New Jersey, 2010)
3. AF Mindikoglu, A-J van der Veen, in Proc. IEEE Int. Conf. Acoustics, Speech and Signal Processing 2008. Separation of overlapping RFID signals by antenna arrays, Las Vegas, USA), pp. 2737-2740. 31 Mar. - 4 Apr. 2008

4. MA Ingram, MF Demirkol, D Kim, in Int. Symp. Signals, Systems, and Electronics. Transmit diversity and spatial multiplexing for RF links using modulated backscatter, (Tokyo, Japan). 24-27 July 2001

5. JD Griffin, GD Durgin, in Proc. 2009 IEEE Int. Conf. RFID. Multipath fading measurements for multi-antenna backscatter RFID at $5.8 \mathrm{GHz}$, (Orlando, USA), pp. 322-329. 27-28 Apr. 2009

6. C He, ZJ Wang, in Proc. 23rd Canadian Conf. Electrical and Computer Engineering. Gains by a space-time-code based signaling scheme for multiple-antenna RFID tags, (Calgary, Canada), 2-5 May 2010

7. D-Y Kim, H-S Jo, H Yoon, C Mun, B-J Jang, J-G Yook, Reverse-link interrogation range of a UHF MIMO-RFID system in Nakagami-m fading channels. IEEE Trans. Industrial Electronics. 57, 1468-1477 (2010)

8. C Angerer, R Langwieser, G Maier, M Rupp, in IEEE 2009 Int. Microwave Workshop on Wireless Sensing, Local Positioning, and RFID. Maximal ratio combining receivers for dual antenna RFID readers, (Cavtat, Croatia), 24-25 Sept. 2009

9. R Langwieser, C Angerer, AL Scholtz, in Proc. IEEE 2010 Radio and Wireless Symp. A UHF frontend for MIMO applications in RFID, (New Orleans, USA), pp. 124-127. 10-14 Jan. 2010

10. C Angerer, R Langwieser, M Rupp, RFID reader receivers for physical layer collision recovery. IEEE Trans. Commun. 58, 3526-3537 (2010)

11. M Mi, MH Mickle, C Capelli, H Switf, RF energy harvesting with multiple antennas in the same space. IEEE Antennas Propagation Mag. 47(5), 100-106 (2005)

12. VTarokh, H Jafarkhani, AR Calderbank, Spacetime block codes from orthogonal designs. IEEE Trans. Inform. Theory. 45, 1456-1467 (1999)

13. T Kaiser, F Zheng, Ultra Wideband Systems with MIMO. ((Wiley, Chichester, 2010)

14. F Zheng, T Kaiser, in 4th Int. EURASIP Workshop on RFID Technology (RFID 2012). On the transmit signal design at the reader for RFID MIMO systems, Turin, Italy), 27-28 Sept. 2012

doi:10.1186/1687-3963-2012-9

Cite this article as: Zheng and Kaiser: A space-time coding approach for RFID MIMO systems. EURASIP Journal on Embedded Systems 2012 2012:9.

\section{Submit your manuscript to a SpringerOpen ${ }^{\mathcal{O}}$ journal and benefit from:}

- Convenient online submission

- Rigorous peer review

- Immediate publication on acceptance

- Open access: articles freely available online

- High visibility within the field

- Retaining the copyright to your article

Submit your next manuscript at $>$ springeropen.com 